\title{
Alexithymia and Empathy in a Non- Clinical Population: How do they Correlate?
}

\begin{abstract}
Alexithymia and empathy are functional concepts surrounding human emotions.

This study aimed to estimate the association between alexithymia and empathy within a neurotypical population.

The study was a cross sectional survey conducted within a nonclinical population of medical students at a University in England using voluntary sampling to complete the Toronto Alexithymia Scale (TAS), Basic Empathy Scale (BES), General Health Questionnaire- 12.
\end{abstract}

Alexithymia and empathy scores did not show a statistically significant correlation. There was a statistically significant negative correlation between total alexithymia and cognitive empathy scores (correlation co-efficient was -0.184 , $\mathrm{p}$ value was 0.013 ). Men and women differed significantly on empathy scores with women showing significantly higher empathy.

The relationship between the understanding of one's own emotions and the interpretation of others' emotions are different functions with a more complex interaction than a simple linear correlation. Future research should focus on further exploring the differences between cognitive and affective empathy.

Keywords: Alexithymia, Empathy, Cognitive, Affective, Emotion

\section{Defining Alexithymia}

Alexithymia is a term derived from Ancient Greek words that literally mean "without words for emotions". It was coined [1] to describe people who appeared to have deficiencies in understanding, processing, or describing their emotions. Empathy is also derived from a Greek word which means physical affection or partiality. It is commonly defined as one's ability to recognize, perceive and directly feel the emotions of another.

\section{Alexithymia as a concept}

Alexithymia is considered to be a personality trait that places individuals at risk for other medical and psychiatric disorders while reducing the likelihood that these individuals will respond to conventional treatments for the other conditions. [2] It is considered to be a personality trait that varies in severity from person to person. A person's alexithymia score can be measured with questionnaires such as the Toronto Alexithymia Scale (TAS20) [3], the Bermond-Vorst Alexithymia Questionnaire (BVAQ) [4] or the Observer Alexithymia Scale (OAS).[2]
Nandini Chakraborty*1, Harry

Mehmet $^{2}$ and Traolach Brugha ${ }^{3}$

1 MD, FRPsych, Consultant

Psychiatrist, Leicestershire

Partnership NHS Trust

PIER team, Merlyn Vaz Health and

Social Care centre, Leicester,

Honorary Professor, University of

Leicester, Department of Health

Sciences

2 Honorary Assistant Psychologist, Leicestershire Partnership Trust

${ }^{3}$ MD (NUI), FRCPsych, SFHEA,

Professor of Psychiatry, Social and Epidemiological Psychiatry Research Group, Department of Health Sciences, University of Leicester

Correspondence:

Nandinichakraborty@doctors.org.uk

Cite as: Chakraborty, N., Mehmet, H. \& Brugha, T. (2021) Alexithymia and Empathy in a Non-Clinical Population: How do they Correlate? The Physician Vol 7; Issue 2: 1-8 Dec'21 DOI: https://doi.org/10.38192/1.7.2.1

Article Information

$\begin{array}{ll}\text { Submitted } & 26.09 .2021 \\ \text { Revised } & 30.10 .2021 \\ \text { Published } & 01.12 .2021\end{array}$

ISSN 2732-513X (Print)

ISSN 2732 - 5148 (Online)

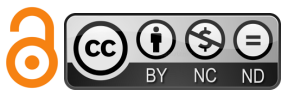


Alexithymia is defined by Taylor:[5]

- Difficulty identifying feelings, distinguishing between feelings and the bodily sensations of emotional arousal,

- Difficulty describing feelings to other people,

- Constricted imaginal processes, as evidenced by a paucity of fantasies,

- A stimulus-bound, externally oriented cognitive style.

It is also suggested [3] that there may be two kinds of alexithymia, 'primary alexithymia' which is an enduring psychological trait which does not alter over time, and 'secondary alexithymia' which is state dependent and disappears after the evoking stressful situation has changed. These two manifestations of alexithymia are otherwise called 'trait' or 'state' alexithymia.

It was noted [6] that patients with alexithymia often mentioned like anxiety or depression. The distinguishing factor was their inability to elaborate beyond a few limited adjectives such as "happy" or "unhappy" when describing these feelings. The core issue is that alexithymics have poorly differentiated emotions limiting their ability to distinguish and describe them to others. This contributes to the sense of emotional detachment from themselves and difficulty connecting with others, making alexithymia negatively associated with life satisfaction even when depression and other confounding factors are controlled for.

\section{Empathy as a concept}

The notion of "Einfühlung" was theoretically developed in nineteenth- and early twentiethcentury German aesthetics especially by Robert Vischer and Theodor Lipps. [7] The German notion of "Einfühlung", was translated as 'empathy' by Edward Titchener and James Ward, thereby introducing the term into the English language. Empathy is one's ability to recognize, perceive and directly feel the emotion of another.

There are two major aspects of empathy:

- Cognitive empathy- the intellectual/imaginative apprehension of another's mental state. (Theory of mind)
- Emotional empathy- an emotional response to the emotional responses of others. (Affective empathy)

It could be an intuitive assumption that alexithymia would directly correlate with a lack of empathy in an individual. There are studies in clinical populations which have directly presumed this correlation. [8] The concept of emotional intelligence [9] combines the concepts of self-awareness (the lack of which can be construed as alexithymia) with social awareness (empathy). Alexithymia and the lack of empathy overlap in a number of clinically relevant areas including autism spectrum disorders, psychopathy and personality disorders. Both concepts are also found important in violence, aggression and response to psychotherapy.[8,10]

Empathy can be cognitive and affective. [11] Affective empathy requires cognitive awareness of the emotions of another, but it appears that cognitive awareness is possible without an affective component. Although studies have looked at both concepts in similar clinical populations, detailed study within normal populations is lacking.

Thereby our study's aim was:

- to examine the relationship between alexithymia and empathy within a normal population.to establish whether there is a negative correlation between the scores of alexithymia and empathy within a non-clinical population, to estimate the magnitude of the correlation.

Given previous research, it was hypothesised that there would be significant differences in total empathy scores between males and females. [12]

\section{Method}

The study design was a cross sectional survey conducted within a non-clinical population with voluntary sampling.

The study participants were students at an English medical school.

A GHQ-12 was included within the questionnaire as a screen and anyone scoring above 36 was excluded from the analysis. 
Students were also advised in writing that if the completion of the forms caused them significant distress, they were to contact their general practitioner, or the student counselling Service.

One of the authors NC attended several lectures, for medical students and explained the proposed study to them. Questionnaires were then left in the classroom to be picked up by students who were willing to fill them in.

A sealed cardboard box was provided for questionnaires to be deposited in. A covering letter in the beginning of the questionnaire with NC's contact details and relevant details of the study was provided for the students to keep. An information and consent form was attached (but not stapled) to the questionnaire. Participants were asked to detach consent forms from the filled in questionnaires before depositing them in designated cardboard boxes, so that all answers are anonymised, but signed consent was obtained from the participants. This process was repeated with a number of student batches, until NC collected 215 questionnaires.

Ethical approval was applied for and granted from the Medical School where the students were based.

The questionnaire contained sociodemographic details such as gender, age, level of education using the following scales:

\section{Toronto alexithymia scale (TAS-20)}

The TAS-20 is a self-report scale that is comprised of 20 items. Items are rated using a 5 -point Likert scale whereby $1=$ strongly disagree and $5=$ strongly agree. There are 5 items that are negatively keyed (items 4, 5, 10, 18 and 19). The total alexithymia score is the sum of responses to all 20 items. The TAS-20 uses cut-off scoring: equal to or less than $51=$ non-alexithymia, equal to or greater than $61=$ alexithymia. Scores of 52 to $60=$ possible alexithymia. Studies have demonstrated that the internal consistency of the total TAS scale, as well as the 8-month retest reliability, are adequate. [13] The TAS correlates fairly well with an interview-based evaluation of alexithymic features.

\section{Basic Empathy Scale (BES)[14]}

The Basic Empathy scale is a 20-point selfreport questionnaire that scores items on a five- point Likert Scale from 'strongly disagree to strongly agree'. A scoring key denotes whether each item is to be scored positively or negatively. Eight items are scored negatively and once these scores are reversed; all the items can be added up to give a total empathy score. Nine items are added to produce the cognitive empathy score and eleven items are added to produce the affective empathy score. Internal consistency (alpha) estimates for BES range from 0.79 to 0.85 . Confirmatory factor analysis has been used to support a two-factor structure. Consistent with prior research, there are significant differences between male and female scores on the BES. BES scores are also found to significantly correlate with measures of intelligence, extraversion, agreeableness conscientiousness, and openness.

\section{General Health Questionnaire- 12[15]}

The General Health Questionnaire (GHQ) is a measure of current mental health. The questionnaire was originally developed as a 60item instrument but at present a range of shortened versions of the questionnaire including the GHQ-30, the GHQ-28, the GHQ-20, and the GHQ-12 is available. The scale asks whether the respondent has experienced a particular symptom or behaviour in the last four weeks. Each item is rated on a four-point scale (less than usual, no more than usual, rather more than usual, or much more than usual) which is scored on a Likert Scale of 1 to 4. A score of more than 36 according to the GHQ manual was the cut-off point.

It has been found to have high internal consistency and good retest reliability. [16] Its validity has been proved by its linear associations with independent clinical assessments and it has been shown to have good discriminatory power. [15]

\section{Analysis}

Due to the non-normal distribution of the questionnaire scores (e.g., cognitive empathy (Shapiro-Wilk value, $\mathrm{p}=0.016$ ), a nonparametric test was used to assess correlations (Spearman).

In order to analyse the relationship between alexithymia and empathy, the scores on the Basic Empathy Scale-26 were separated into cognitive and affective elements by totalling each respective score to produce a total for each element. [14] These elements were then 
included alongside the overall total empathy scores for correlational analysis with total alexithymia scores from the Toronto Alexithymia Scale $(n=180)$.

The difference in mean scores between those scoring positive for alexithymia (TAS score $>=74$ ) and those scoring negative for alexithymia (TAS score $<=62$ ), and their respective total empathy scores was estimated. The assumptions for parametric testing could not be satisfied, hence the use an appropriate alternative, the Mann Whitney U test.

As predicted by our hypothesis, an additional analysis was performed to determine if there was a significant difference in empathy scores between genders (male/female).

\section{Results}

The original sample size was 215 students. Seven questionnaires were incomplete, leaving 208 for analysis. Those scoring > 36 on the GHQ12 were removed, which totalled 17 participants. An additional 11 participants were removed once they were identified to be outliers for the variables that were intended to be analysed, resulting in a final number for analysis, $n=180$. [17]

$42 \%$ of the sample was male and $58 \%$ female. $68 \%$ were less than 25 years of age. $59 \%$ held A level qualifications while $41 \%$ held graduate qualifications. (see Table 1).

Performing a Spearman's Rank correlational analysis determined the following relationships between variables (Table 3). There was a very weak negative correlation between total alexithymia scores and total empathy scores, which was not statistically significant. There was a negative correlation between total alexithymia scores and total scores for cognitive empathy which was statistically significant. There was a weak positive correlation between total alexithymia scores and total affective empathy scores which was not statistically significant.

Finally, when considering gender, a MannWhitney $U$ test indicated that the empathy scores (BES) were greater for females ( median=79; mean rank $=105.08)$ than for males (median $=74$; mean rank $=70.55)$, with a statistically significant value, $\mathrm{U}=2435.50$ ( $\mathrm{Z}=-$ 4.39 ), $\mathrm{p}<0.001$, with a medium effect size ( $\mathrm{r}=-$ 0.32). (Table 2)

\section{Discussion}

Our study found that there was a weak positive correlation between alexithymia and affective empathy scores; however, this was not statistically significant. There was a statistically significant negative correlation between alexithymia scores and cognitive empathy. This is like the findings from [18] Shah et.al. which is the first study to detect a relationship between alexithymia and low cognitive empathy even after controlling for autism and affective empathy.

\section{Alexithymia and empathy studied in normal population}

Shah et al [18] studied autistic traits, empathy and alexithymia in large samples of general population. They demonstrated through multiple regression analyses that both trait autism and alexithymia were uniquely associated with atypical empathy, but dominance analysis found that trait autism, compared to alexithymia, was a more important predictor of atypical cognitive, affective, and overall empathy. Together, these findings indicated that atypical empathy in ASD is not simply due to co-occurring alexithymia. The authors also demonstrated findings on affective empathy which were novel but inconsistent. The first regression analysis unexpectedly showed that alexithymia, but not autism, was associated with high affective empathy whilst controlling for cognitive empathy. However, the second regression analysis failed to replicate this finding, instead showing that autism, not alexithymia, was associated with high affective empathy.

\section{Alexithymia and empathy studied in clinical populations}

Ziermans et al in their 2018 study [19] demonstrated that students with autistic spectrum disorder showed higher cognitive alexithymia and lower cognitive empathy compared to neurotypical students. The groups did not vary on affective empathy or total scores of alexithymia. Hence, they concluded that intellectually advanced individuals with autism spectrum disorder experience serious impairments in the cognitive processing of social-emotional information.

Rueda et al in 2015 [11] also demonstrated that young people with autistic spectrum disorder scored lower than controls on cognitive 
empathy but scored within the average range on affective empathy.

Both these studies thereby show disconnect between cognitive empathy and affective empathy, similar to what our study and Shah et al [18] have demonstrated.

Males and females differed significantly in their empathy scores with females demonstrating greater empathy than males. This is consistent with findings reported by Toussaint and Webb, 2005.[12]

However, this study depended on the selfreport questionnaires and the sample was taken from a very specific group i.e. medical students. Therefore, it would be useful to expand future studies to include a more diverse sample and include face to face interviews.

\section{Conclusions}

Our study adds to the existing body of literature, which shows a significant negative correlation between alexithymia and cognitive empathy but not affective empathy in both clinical and non-clinical populations. This demonstrates that cognitive and affective empathy are potentially independent functions with cognitive empathy having a closer relationship with alexithymia than affective empathy.

Our study similarly demonstrates cognitive empathy to be a particular area which is affected with higher alexithymia scores. This might be of clinical significance. The question arises- could there be a focus on helping individuals with conditions in which there is a higher alexithymia, such as autistic spectrum disorder to cognitively recognise emotions of other people by tapping into their affective responses which may be less deficient compared to the cognitive response?

Overall, our findings reveal that the relationship between the understanding of one's own emotions and interpretation of others' emotions are possibly different functions with a more complex interaction than simple correlation in a linear manner. Interpreting others' emotions in an emotional (affective) and logical (cognitive) way appear to function differently opening possibilities of further research to explore how these emotional functions could interact with each other and how this could potentially impact on psychological and behavioural therapies.

The findings of this study suggest the feasibility and need to replicate the study in patient populations especially those with an autistic spectrum disorder which could have further clinical implications for understanding emotions and planning clinical interventions in autistic spectrum disorder.

\section{Declaration of interest \\ None}

\section{Acknowledgements}

The authors are grateful to Dr D Jollife for the permission and guidance to use the Basic Empathy Scale. 
Table 1: Demographic characteristics

\begin{tabular}{|c|c|c|c|}
\hline $\begin{array}{c}\text { Demographics } \\
(\mathbf{n = 1 8 0})\end{array}$ & Number & $\mathbf{\%}$ \\
\hline Gender & Male & 76 & 42 \\
\hline Age & Female & 104 & 58 \\
\hline & $<25$ & 122 & 68 \\
\hline Qualifications & $>25$ & 58 & 32 \\
\hline & A levels & 106 & 59 \\
\hline & $\begin{array}{c}\text { Graduate } \\
\text { qualification }\end{array}$ & 74 & 41 \\
\hline
\end{tabular}

Table 2: Descriptive statistics of emotional variables

\begin{tabular}{|c|c|c|c|}
\hline Variable & Mean Score & $\begin{array}{c}\text { Male- } \\
\text { mean (range) }\end{array}$ & $\begin{array}{c}\text { Female- } \\
\text { mean (range) }\end{array}$ \\
\hline Empathy & 77 & $73.8( \pm 33)$ & $78.8( \pm 37)^{*}$ \\
\hline Alexithymia & 63 & $62.4( \pm 41)$ & $63.5( \pm 42)$ \\
\hline Cognitive empathy & 36 & $35.6( \pm 16)$ & $37( \pm 28)$ \\
\hline Affective empathy & 40 & $41.8( \pm 19)$ & $41.8( \pm 24)$ \\
\hline GHQ & 24 & $24( \pm 22)$ & $24.2( \pm 22)$ \\
\hline
\end{tabular}

Table 3: Correlation results

\begin{tabular}{|c|c|c|c|c|}
\hline & & $\begin{array}{c}\text { EMPATHY } \\
\text { TOTAL ON THE } \\
\text { BES SCALE }\end{array}$ & $\begin{array}{c}\text { COGNITIVE } \\
\text { EMPATHY }\end{array}$ & $\begin{array}{c}\text { AFFECTIVE } \\
\text { EMPATHY }\end{array}$ \\
\hline $\begin{array}{c}\text { ALEXITHYMIA } \\
\text { TOTAL } \\
\text { (TAS-20) }\end{array}$ & $\begin{array}{c}\text { Correlation } \\
\text { Coefficient }\end{array}$ & -0.067 & -0.184 & 0.003 \\
\hline & $\begin{array}{c}\text { Sig. (2-tailed, } \\
\text { p-value) }\end{array}$ & 0.375 & $0.013^{*}$ & 0.971 \\
\hline & $\mathrm{N}$ & 180 & 180 & 180 \\
\hline
\end{tabular}

* Statistically significant $(\mathrm{p}<0.05)$ 


\section{References:}

1 Sifneos PE. The Prevalence of 'Alexithymic' Characteristics in Psychosomatic Patients. PPS 1973;22:255-62. doi:10.1159/000286529

2 Haviland MG, Warren WL, Riggs ML. An observer scale to measure alexithymia. Psychosomatics 2000;41:385-92. doi:10.1176/appi.psy.41.5.385

3 Taylor GJ, Bagby RM, Ryan DP, et al. Criterion validity of the Toronto Alexithymia Scale. Psychosom Med 1988;50:500-9. doi:10.1097/00006842198809000-00006

4 de Vroege L, Emons WHM, Sijtsma K, et al. Psychometric Properties of the BermondVorst Alexithymia Questionnaire (BVAQ) in the General Population and a Clinical Population. Front Psychiatry 2018;9:111. doi:10.3389/fpsyt.2018.00111

5 Taylor GJ, Bagby RM, Parker JDA. Disorders of Affect Regulation: Alexithymia in Medical and Psychiatric Illness. Cambridge: : Cambridge University Press 1997. doi:10.1017/CB09780511526831

6 Sifneos PE. Anxiety suppressive psychotherapy: Teaching to anticipate confusing situations. Psychotherapy and Psychosomatics 1967;15:63-63.

7 Ganczarek J, Hünefeldt T, Olivetti Belardinelli M. From "Einfühlung” to empathy: exploring the relationship between aesthetic and interpersonal experience. Cogn Process 2018;19:141-5. doi:10.1007/s10339-018-0861-X

8 Empathy and Judging Other's Pain: An fMRI Study of Alexithymia | Cerebral Cortex | Oxford Academic. https://academic.oup.com/cercor/article /17/9/2223/273662 (accessed 1 Dec 2021).

9 Goleman D. Emotional Intelligence: 25th Anniversary Edition. 1st edition. Bloomsbury Publishing 2020.

10 Decety J. Empathy in Medicine: What It Is, and How Much We Really Need It. Am J Med 2020;133:561-6.

doi:10.1016/j.amjmed.2019.12.012
11 Dissociation between cognitive and affective empathy in youth with Asperger Syndrome: European Journal of Developmental Psychology: Vol 12, No 1. https://www.tandfonline.com/doi/abs/1 $0.1080 / 17405629.2014 .950221$ (accessed 2 Dec 2021).

12 Toussaint L, Webb JR. Gender differences in the relationship between empathy and forgiveness. J Soc Psychol 2005;145:67385. doi:10.3200/SOCP.145.6.673-686

13 Kauhanen J, Julkunen J, Salonen JT. Validity and reliability of the Toronto Alexithymia Scale (TAS) in a population study. J Psychosom Res 1992;36:687-94. doi:10.1016/0022-3999(92)90058-a

14 Jolliffe D, Farrington DP. Development and validation of the Basic Empathy Scale.J Adolesc 2006;29:589-611. doi:10.1016/j.adolescence.2005.08.010

15 GHQ - General Health Questionnaire. https://eprovide.mapitrust.org/instruments/general-healthquestionnaire (accessed 2 Dec 2021).

16 Werneke U, Goldberg DP, Yalcin I, et al. The stability of the factor structure of the General Health Questionnaire. Psychol Med 2000;30:823-9. doi:10.1017/s0033291799002287

17 Kwak SK, Kim JH. Statistical data preparation: management of missing values and outliers. Korean J Anesthesiol 2017;70:407-11.

doi:10.4097/kjae.2017.70.4.407

18 Trait Autism is a Better Predictor of Empathy than Alexithymia | SpringerLink. https://link.springer.com/article/10.100 7/s10803-019-04080-3 (accessed 2 Dec 2021).

19 Impairments in cognitive empathy and alexithymia occur independently of executive functioning in college students with autism - Tim Ziermans, Ymke de Bruijn, Renee Dijkhuis, Wouter Staal, Hanna Swaab, 2019. https://journals.sagepub.com/doi/10.117 7/1362361318817716 (accessed 2 Dec 2021). 